\title{
Abstraction Layer For Standardizing APIs of Task-Based Engines
}

\author{
Rabab Alomairy, Hatem Ltaief, Mustafa Abduljabbar, and David Keyes
}

\begin{abstract}
We introduce AL4SAN, a lightweight library for abstracting the APIs of task-based runtime engines. AL4SAN unifies the expression of tasks and their data dependencies. It supports various dynamic runtime systems relying on compiler technology and user-defined APIs. It enables an application to employ different runtimes and their respective scheduling components, while providing user-obliviousness to the underlying hardware configurations. AL4SAN exposes common front-end APIs and connects to different back-end runtimes. Experiments on performance and overhead assessments are reported on various shared- and distributed-memory possibly hardware accelerator-equipped systems. A range of workloads, from compute-bound to memory-bound regimes, are employed as proxies for current scientific applications. The low overhead (less than $10 \%$ ) achieved using a variety of workloads enables AL4SAN to be deployed for fast development of task-based numerical algorithms. More interestingly, AL4 SAN enables runtime interoperability by switching runtimes at runtime. Blending runtime systems permits to achieve a twofold speedup on a task-based generalized symmetric eigenvalue solver, relative to state-of-the-art implementations. The ultimate goal of AL 4 SAN is not to create a new runtime, but to strengthen co-design of existing runtimes/applications, while facilitating user productivity and code portability. The code of AL4SAN is freely available at https://github.com/ecrc/al4san, with extensions in progress.
\end{abstract}

Index Terms-Task-Based Programming Model, Dynamic Runtime Systems, Abstraction Layer, API Standardization, User Productivity, LLVM Compiler Infrastructure, Runtime Interoperability.

\section{INTRODUCTION}

Task-based programming models have become ubiquitous in scientific computing. For the last decade, they have demonstrated how they can leverage performance from the bottom of the software stack with numerical libraries [1][7] all the way up to computational simulations and applications [8]-[11]. Thanks to the fine-grained computations, task-based numerical libraries and applications have proven their ability to reduce idle time due to load imbalance, hiding data movement with computations and weakening artifactual synchronization points between processing units. Although they are capable of mitigating many overheads, they rely on dynamic engines or runtime systems to abstract the underlying hardware complexity from end-users. These runtime systems efficiently marshal task data dependencies and schedule the corresponding computational kernels on available hardware resources. There exists a myriad of dynamic runtime systems to support task-based programming models on shared- and distributed-memory systems, possibly equipped with hardware accelerators [12]. The lack of API standardization makes it cumbersome for task-based applications and library developers to exploit different runtimes and their respective features. This requires changes into the original code to port it to a specific task-based engine in order to execute on a given hardware system.

To enhance user productivity, especially with the emergence of challenging massively parallel systems, this paper describes the implementation of AL4SAN, an abstraction

- The authors are with the Extreme Computing Research Center, Computer,Electrical, and Mathematical Sciences and Engineering Division (CEMSE),King Abdullah University of Science and Technology (KAUST), Thuwal 23955-6900, Saudi Arabia. E-mail: Rabab.Omairy, Hatem.Ltaief, Mustafa.Abduljabbar, David.Keyes@kaust.edu.sa.

Manuscript received September 2, 2019; revised September X, 2019. layer library for standardizing APIs of task-based engines. This portable, lightweight software solution enables end users to write once their task-based codes using a collection of AL4SAN's APIs, which unifies the expression of tasks and their data dependencies adopted by the following runtime systems: QUARK [13], St arPU [14], and OpenMP [15]. Among many others, these runtimes have been selected as proxies, both for compiler-based infrastructure technology and userdefined API approaches. AL4SAN features a second level of abstraction and closes the gap between the runtime systems and the applications. It provides a single frontend API to queue tasks and several backends to hook on the appropriate engine. AL4SAN ultimately enables a single-code application to assess various runtimes and their respective scheduling components (e.g., priority, locality, etc.), while providing the user-obliviousness to the underlying complex hardware configurations.

The idea of AL4SAN originated from our observation that task-based runtime systems are not yet mature, and that standardization is usually a gradual process. For instance, following OmpSs' early adoption [16], it took five years for OpenMP to support task constructs with inter-task data dependencies (from v3.0 [17] to v4.0 [15]). Nevertheless, this slow process has positive implications. Indeed, multiple tasking "swim lanes" facilitate the convergence toward a lasting maturity. Multiple lanes also permit to pursue critical features' development that may not yet be suitable for standardization. The goal of AL4SAN is not to create "yet another runtime system" and having to deal with its complex internal scheduling function. Developed as a thin layer of abstraction, its goal is to help end users focus on strengthening runtime/application co-design instead, while building synergism to enhance user productivity and code portability in tandem with the lower level existing task- 
based runtime systems. Indeed, AL4SAN may even be used as an efficient tool to further broaden the hardware support for existing task-based numerical algorithms. In particular, we demonstrate how AL4SAN enables runtime interoperability for the sake of combining specific algorithmic features from different task-based numerical libraries. By switching runtimes at runtime, AL4SAN is able to deploy the task-based generalized symmetric eigenvalue solver, a critical numerical solver especially for material science applications, onto heterogeneous hardware configurations for the first time.

Last but not least, the overhead of the additional software layer engendered by AL4SAN is limited to reproduce reasonable performance fidelity of the native application with the corresponding supported runtime. To assess this overhead, we benchmark AL4SAN with the Chameleon [18] and HiCMA [19], [20] task-based numerical libraries, used as representative compute-bound and memory-bound workloads of current large-scale scientific applications, respectively. The reported overhead turns out to be acceptable (less than $10 \%$ ) on various hardware system configurations. AL4SAN's abstraction layer may always be deactivated once the adequate runtime backend has been identified in order to remove transient overheads altogether. A source to source compiler tool may be implemented to ease the translation from AL4SAN to the selected native runtime's API but this is beyond the scope of the paper. The code of AL4SAN is freely available ${ }^{1}$ and under active development for broader runtime supports.

The remainder of the paper is as follows. Section 2 presents the rich literature of task-based programming models associated with runtime systems and highlights the lack of API standardization. Section 3 introduces the design of AL4SAN software infrastructure. Section 4 briefly describes the compute-bound and memory-bound workloads for assessing the overhead of AL 4 SAN. Section 5 highlights the implementation of AL4SAN and identifies common and flexible APIs for making runtime-agnostic task-based applications. Section 6 describes how to further leverage AL4SAN's capabilities by relying on runtime interoperability. Section 7 analyzes the performance and the overhead of AL4SAN on aforementioned workloads and demonstrates the effectiveness of our lightweight software layer. Section 8 summarizes the contributions and describes planned work.

\section{Related Work}

The number of engines that inherently support task-based programming models has been dramatically increasing in the last decade. This is mostly due to the emergence of compiler-based technologies that are usually embedded into domain specific languages, such as, to cite a few, Charm++ [21], Legion [22] Uintah [11], and Kokkos [23]. Some of these runtimes have enabled scientific applications to scale up and reach petascale performance on today's fastest supercomputers.

There are also runtimes using pragma-based tasking approaches, such as Ompss [16], [24], [25], which have eventually been integrated into the OpenMP standard specifications [17], and later in [15] with inter-task dependencies.

1. https://github.com/ecrc/al4san
Moreover, there exist many dynamic runtime systems, which rely on high-level user-defined APIs, and make them portable across various software and hardware configurations, such as StarPU [14], QUARK [13], and Parallex [26]. They are capable of scheduling complex Directed Acyclic Graphs (DAGs), whose nodes represent computational tasks whereas edges define the data dependencies, while leveraging applications' performance on multiple hardware systems.

Given the wide spectrum of task-based engines, much effort has been made to standardize the various runtime systems, by abstracting their APIs or building compiler solutions with language constructs [27]-[29]. This effort turns out to be extremely challenging, due to the heterogeneity of the runtimes' frontends/APIs, their different levels of support on a given hardware/software system configuration, and the broad range of scheduling features.

With regards to related works, the Kokkos and Raja projects use pragmas and therefore, rely on compiler technologies. Their tasking approach is confined within the scope of data-level parallelism brought to the fore by the fork-join paradigm. The approach of AL4SAN is broader, since it also supports queues and runs tasks defined in nested and/or subsequent loops. The inter-task dependencies across loops are then tracked by the corresponding runtime. We have also looked at the HiHAT [30] and DARMA [29] on-going projects, which have some commonalities with AL4SAN in abstracting backend runtimes.

In this paper, we introduce and implement AL4SAN with support initially for three dynamic runtime systems, based on the following rationale: StarPU [14] and QUARK [13] as representatives of task-based engines relying on userdefined APIs, and OpenMP [15] as the de facto standard programming model based on compiler technology. While this current list of supported runtimes is not exhaustive, it still enables to design and implement AL4SAN in a holistic way with a progressive and widening scope moving forward. Last but not least, AL 4SAN has been successfully integrated into Chameleon [18] and HiCMA [19], i.e., software packages which support dense and data-sparse linear algebra workloads, respectively. AL4SAN may play a key role in facilitating the development and deployment of future numerical algorithms based on task-based programming models to express fine-grained parallelism.

\section{AL 4 SAN SOFTWARE INFRASTRUCTURE}

Written in the $C$ programming language, the abstraction layer of AL4SAN features a unified, lightweight API that maps to multiple backends (i.e., StarPU, QUARK, and OpenMP). On top of a specialized backend, a single frontend allows the user program to define tasks that may run on various hardware systems (e.g., CPUs and/or GPUs), and to express data dependencies between these tasks. These twolayer APIs bridge the application's and runtime's spaces, as demonstrated in Figure 1. The herein studied task-based runtimes provide support for features that typically include initializing the runtime scheduler, adding tasks to the queue, marshaling sequence of tasks, looking for predefined function declaration, unpacking the data structures to retrieve data, waiting at a synchronization point, and eventually, 


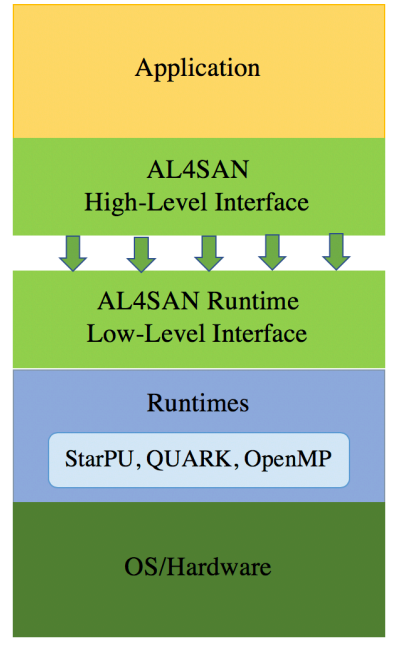

Fig. 1: AL4SAN structure.

terminating the scheduler. From these features, common interfaces across runtimes have been identified and constitute the inherent AL4SAN APIs.

Before delving into the technical description of AL4SAN APIs, the support for OpenMP requires special treatment since its programming model exposes parallelism via preprocessor directives, which can be resolved only during compilation time. This is not possible as it natively stands, since AL4SAN needs to resolve tasks and their data dependencies' constructs at runtime. A possible workaround is to use sophisticated preprocessing metaprogramming (e.g., the Boost preprocessing library), at the expense of limitations due to the supported number of dependencies and possibly the corresponding significant compilation time, which may defeat the lightweight purpose of AL4SAN. The alternative embraced herein, however, is to control the OpenMP runtime library by using LLVM, which grants AL 4 SAN more flexibility by directly accessing low-level OpenMP APIs. A similar approach can be applied to eventually support OmpSs [16], [24] by using the low-level APIs of the intermediate representations generated from source-to-source compilations.

AL4SAN APIs can be categorized by three main building blocks: initialization, task management, and termination. The initialization API encompasses the creation of the context data structure and its running environment via the setup function, as defined for each runtime. This context holds the runtime scheduler and its status during the execution. Once the underlying scheduler is initialized, AL4SAN provides abstraction of internal functions to create the data structure for the context. This data structure encapsulates the sequence handle returned from the corresponding runtime, which is responsible for managing execution stream of task groups. Each sequence handle is, therefore, attached to a single context, which enables multiple contexts to interoperate on the same application. For instance, multiple contexts and their respective sequence handles may be useful for divide-and-conquer workloads. The second building block is the task management, which involves task insertion with packing/unpacking functions. This is where AL 4 SAN conveys information about the task types (e.g., CPU or GPU kernels) as well as their data dependencies (e.g., input, output, inout), along with a list of arguments (e.g., scheduling priorities) to the specific backend. The final stage of AL4SAN's workflow is termination. Once the execution has finished, AL4SAN provides a common API to terminate existing sequence and to shut down the runtime through its appropriate API.

\section{Workload Characterization}

This section describes the compute-bound and memorybound workloads used to evaluate the overhead introduced by AL 4 SAN abstraction layer. These types of workloads may resemble the application spectrum at both ends of performance categories. For compute-bound workloads, the taskbased dense matrix-matrix multiplication kernel (GEMM) and Cholesky factorization have been selected as representative workloads with high arithmetic intensities. Such workloads are compute intensive and may permit to even hide the overhead of the task-based engines itself. These two dense matrix operations are implemented based on tile algorithms within the Chameleon library [18], a state-ofthe-art dense linear algebra (DLA) software package using task-based programming models. Chameleon relies on various runtimes, including those supported by AL4SAN, i.e., StarPU, and QUARK.

Furthermore, the algorithmic development of low-rank matrix approximation (LRMA) has gained lots of interest [31]. The idea consists in exploiting the data sparsity of the dense matrix, by approximating off-diagonal blocks up to an error threshold. Therefore, LRMA permits to handle large dense matrix sizes, thanks to a lower memory footprint. While LRMA also reduces the algorithmic complexity allowing a faster time to solution, it pushes the arithmetic intensity of native dense matrix compute-bound operations toward the memory-bound regime of execution, where data movement and bus bandwidth saturation become the main bottlenecks. In particular, the Hierarchical Computations on Manycore Architectures framework [19] (HiCMA) provides LRMA support for the Cholesky factorization on datasparse matrices, using tile low-rank data layout [20]. Similar to Chameleon, HiCMA has been used as a testbed to study the overhead of AL4SAN, but on memory-bound workloads instead.

AL4SAN provides data abstraction to operate on a matrix object (as well as vector and scalar objects) through the definition of a data descriptor. This descriptor expresses the matrix data using a tile structure, similar to the Chameleon [18] and PLASMA [1] libraries. Computational tasks are then mapped to these tiles, which translate the original source code into a dataflow represented by a Directed Acyclic Graph (DAG). This data abstraction is then leveraged by AL4SAN, which in turn abstracts the tasks and the underlying dynamic runtime systems.

\section{Standardizing Runtime APIS}

This section tackles the individual interfaces of AL4SANcompatible runtimes with implementation details, paving the way for defining its API, given the capabilities of the runtimes under consideration. 


\subsection{Description of Existing Runtime APIs}

QUARK. The QUeuing And Runtime of Kernels (QUARK) runtime system is initialized by calling QUARK_New function, which allocates the scheduler's data structure and spawns/manages the worker threads. QUARK scheduler is terminated using QUARK_Delete (quark), which stops the worker threads. In between QUARK_New and QUARK_Delete (quark) calls, QUARK creates and handles the tasks' sequence. Tasks can be added to the QUARK runtime system by means of calling QUARK_Insert_Task function from the master thread. The first three parameters of this function are pointers to: the QUARK data structure, the work function, and task-specific flags such as "priority". The fourth parameter of task insert function is a variadic list (i.e., va_list), which holds a variable length list of arguments describing task's data. Each argument is expressed by a triplet: the size of argument in bytes, a pointer to the argument, and a flag specifying the way that the argument is to be treated. The dependency direction can be either INPUT, OUTPUT, INOUT, VALUE, SCRATCH, and NODEP. When task dependencies are satisfied and it is ready to be executed, QUARK scheduler invokes the corresponding task's work function by probing the declaration of function (Quark * quark). Then, the data is unpacked by QUARK using quark_unpack_args_\# macro, where "\#" indicates the number of arguments to be unpacked.This overall mode of operation, which consists in executing sequential interdependent tasks in parallel, relies on a Sequential Task Flow (STF) model. The corresponding task graph is not known a priori and is discovered only at runtime, while the tasks are being queued by the master thread.

StarPU. The StarPU runtime system is initialized by calling starpu_init function to configure the scheduling policy, the number of cores, etc., using starpu_conf data structure. If MPI is enabled, starpu_mpi_init should be called and starpumpi library must be initialized with the given MPI communicator. To terminate the StarPU runtime system starpu_shutdown is called as well starpu_mpi_shutdown for MPI tasks. Before task creation, a call to starpu_data_register registers the application's data, whereas starpu_codelet defines the codelet. starpu_data_register returns a handle to the data buffer, which facilitates data movement between the accelerator and main memory. In addition, the starpu_codelet is a per-task handle that specifies the host architecture (CPU or GPU), the runtime's buffer count, and codelet's data access modes. At this stage, the task is ready to be queued using starpu_insert_task for shared-memory tasks or starpu_mpi_insert_task for MPI tasks. This task API takes the codelet as first parameter, followed by the argument list, which can be defined either as doublet, containing task options and data buffer, or a triplet, containing data to be copied, too. Task dependencies are expressed using STARPU_R, STARPU_W, and STARP_RW, and task options include STARPU_PRIORITY, STARPU_LOCALITY, and so on. When the task is scheduled for execution, the scheduler calls the work function with the signature function (void *descr[], void * Cl_args). Then, packed data can be extracted from cl_args using starpu_codelet_unpack_args function, while the data buffer can be acquired from descr [] using the STARPU_MATRIX_GET_PTR macro [2], [14]. StarPU also follows the STF model, but the master thread not only queues tasks, but also participates in executing ready tasks whenever possible.

OpenMP. OpenMP is an industry-wide standard for shared memory programming in $\mathrm{C} / \mathrm{C}++$ and Fortran. The OpenMP Architecture Review Board introduced task-based parallelism through the task dependencies extension starting from OpenMP version 4.0. This task model is adopted by the development team of GCC/libGOMP, ICC/libIOMP, and CLANG/libIOMP in their compilers and associated runtime systems. OpenMP uses compiler's directive to describe the task and its data dependencies. Each task is decorated using \#pragma omp task depend directive enclosing a dynamically-scheduled task region. depend specifies the dependency of task shared variables. Data access modes can be expressed by either in, out, or inout. Translating the OpenMP annotated task is similar in GCC, ICC, and CLANG/LLVM compiler. All compilers generate an array list out of the depend clause that contains the addresses of all referenced variables. GCC passes this argument to GOMP_task function generated as a result of the \#pragma omp task directive, while ICC and CLANG passes it to the kmpc_omp_task_with_deps runtime function [32].

Summary of Runtime Features. Table 1 provides a mosaic of the various features supported by the task-based runtime systems studied herein. The table highlights how the levels of support vary significantly from one runtime to another. For instance, QUARK supports only shared-memory systems, while StarPU handles distributed-memory systems equipped with hardware accelerators. However, although StarPU is not the de facto standard task-based engine for scientific applications as the OpenMP runtime, the latter has yet to provide the dynamic resolution of intertask data dependencies between regions. The GPU support with pragmas for OpenMP has been added in the latest 5.0 version, which was not available at the time of development, but will be added in the next AL4SAN software release. Moreover, each runtime provides various heuristics for scheduling (driven by data locality, task priority, work stealing, etc.), which may translate into different performance optimizations. With regards to task management, all runtimes support the sequential task flow (STF), which permits to express a given numerical algorithm using subsequent, and possibly nested loops. STF may improve the development phase for end-users. There are other runtimes under consideration, e.g., PaRSEC [33], which employs an advanced parametrized task graph (PTG) mechanism to express data dependencies. PTG may achieve better performance than STF at large-scale but requires advanced expertise to generate data dependencies along with the help of compiler tools. Further details on the various runtime features are available in the corresponding references. This table, therefore, motivates the importance of abstracting the APIs of these runtimes to enhance user productivity and code portability, as illustrated in the next section.

\subsection{Single Unified API using AL4SAN}

AL4SAN introduces user-callable APIs to unify the various considered runtime APIs for task-based programming. 
TABLE 1: Summary of AL4SAN supported runtimes and their features.

\begin{tabular}{|c|c|c|c|c|c|c|c|}
\hline $\begin{array}{l}\text { Task-based } \\
\text { Runtime }\end{array}$ & $\begin{array}{l}\text { Shared } \\
\text { Memory }\end{array}$ & $\begin{array}{l}\text { Distributed } \\
\text { Memory }\end{array}$ & GPU & Runtime Features & Task Management & $\begin{array}{l}\text { Programming } \\
\text { Interface }\end{array}$ & $\begin{array}{l}\text { Compiler Sup- } \\
\text { port }\end{array}$ \\
\hline $\begin{array}{l}\text { OpenMP } \\
4.0[15]\end{array}$ & $\begin{array}{l}\text { OpenMP } \\
\text { threads }\end{array}$ & $x$ & $x$ & $\begin{array}{l}\text { standard in GCC, ICC, } \\
\text { CLANG, pragma directive }\end{array}$ & $\begin{array}{l}\text { Sequential task flow, } \\
\text { fork-join model }\end{array}$ & C, C++, Fortran & $\sqrt{2}$ \\
\hline StarPU [14] & Pthreads & $\checkmark$ & $\checkmark$ & $\begin{array}{l}\text { prio, dm, dmda, eager } \\
\text { scheduler, work stealing, } \\
\text { priority }\end{array}$ & Sequential task flow & $\mathrm{C}$ & $x$ \\
\hline QUARK [13] & Pthreads & $x$ & $x$ & $\begin{array}{l}\text { priority and locality } \\
\text { hinting, accumulator and } \\
\text { gatherv tasks }\end{array}$ & Sequential task flow & $\mathrm{C}$ & $x$ \\
\hline
\end{tabular}

AL4SAN_Init(runtime, ncpus, ngpus); sequence=AL4SAN_Sequence_Create();

for $k \leftarrow 0$ to $n t$ do

AL4SAN_Insert_Task(AL4SAN_TASK(POTRF), sequence, $\mathrm{A}[\mathrm{k}][\mathrm{k}] \ldots)$;

for $m \leftarrow k+1$ to $n t$ do AL4SAN_Insert Task(AL4SAN_TASK(TRSM), sequence, $\mathrm{A}[\mathrm{k}][\mathrm{k}], \mathrm{A}[\mathrm{m}][\mathrm{k}], \ldots)$;

for $n \leftarrow k+1$ to $n t$ do AL4SAN_Insert_Task(AL4SAN_TASK(SYRK), sequence, $A[n][k], A[n][n], \ldots)$;

for $m \leftarrow n+1$ to $n t$ do

AL4SAN_Insert_Task(AL4SAN_TASK(GEMM), sequence, $\mathrm{A}[\mathrm{m}][\mathrm{k}], \mathrm{A}[\mathrm{n}][\mathrm{k}], \mathrm{A}[\mathrm{m}][\mathrm{n}], \ldots)$;

AL4SAN_Sequence_Wait( sequence);

AL4SAN_Sequence_Destroy(sequence);

AL4SAN_Finalize();

(a) AL4SAN Cholesky factorization.

AL4SAN_Task_CPU(POTRF, POTRF_CPU)

AL4SAN_Insert_Task(AL4SAN_TASK(POTRF), sequence,

AL4SAN_VALUE, \&uplo, sizeof(int),

AL4SAN_VALUE, \&nb, sizeof(int),

AL4SAN_INOUT, A[k][k], AL4SAN_DEP,

AL4SAN_VALUE, \&lda, sizeof(int),

AL4SAN_VALUE, \&info, sizeof(int),

..., ARG_END);

void POTRF_CPU (AL4SAN_arglist *al4san_arg) \{

AL4SAN_Unpack_Arg(al4san_arg, \&uplo, \&nb, \&A, \&lda, \&info );

potrf_(\&uplo, \&nb, A, \&lda, \&info); \}

(b) AL4SAN Task interface.

Fig. 2: AL4SAN's API specification.

AL4SAN provides high-level APIs and low-level APIs encapsulating the implementations of different runtimes. AL 4 SAN initializes its scheduler by calling AL4SAN_Init, which internally calls the corresponding function of the backend. This function is the facade enclosing the runtime-specific initializers (e.g., starpu_init for StarPU engine).

AL4SAN groups tasks using a sequence data structure via AL4SAN_Sequence_Create, which creates and saves the state of the stream of tasks during execution. This function allocates AL4SAN_sequence_t , and it maps to the semantically equivalent version pertaining to the linked runtime (e.g., Quark_Sequence).

To define task and its dependencies information, AL 4 SAN uses AL4SAN_Insert_Task API, which unifies the task insertion API of the backend runtime. The first parameter is AL4SAN_TASK's macro, which accepts "task name" as an argument. This macro prepares work function's invocation by prefixing the runtime's identifier to the specified "task name". The second parameter is the AL4SAN_sequence_t, which owns the sequence handle. The last is an argument triplet encompassing the type of data dependency directions (i.e., AL4SAN_INPUT, AL4SAN_OUTPUT, AL4SAN_INOUT, and AL4SAN_SCRATCH), argument handle, and size of argument in byte.

Figure 2 highlights a task-based pseudocode of the Cholesky factorization (i.e., Figure 2a) using the new unified task API (i.e., Figure 2b). This API has been purposely designed to be close to the currently supported runtimes, to ease integration and adoption. This API is also flexible enough to encapsulate future features and keep track of the latest developments of the supported backend engines.

Once defined, a task's work function can be either a CPU task (AL4SAN_Task_CPU), a GPU task (AL4SAN_Task_GPU), or a heterogeneous task (AL4SAN_Task_CPU_GPU) represented by a macro which takes the task name and a CPU and/or GPU user function. This macro encapsulates work function declaration and the main construct of function's body following the formats depicted by Figure 3. The macro "Frontend" acts like a factory for the "Task Function Declaration" of each runtime. It subsequently assigns the packed data to AL4SAN_arglist, and calls user's function. Upon a user function's entry, data is unpacked using AL4SAN_Unpack_Arg to be retrieved from AL4SAN_arg_list. This function hands over the control to low-level "unpack" routines of each runtime. Finally, the scheduler proceeds to the user application code.

AL4SAN supports several data interfaces to operate on a matrix through AL4SAN_Matrix_Create, on a vector through AL4SAN_Vector_Create, and on a scalar through AL4SAN_Scalar_Create APIs. These interfaces initialize data, allocate memory region if it is not yet specified, and register data in the underlying runtime.

Last but not least, since OpenMP uses preprocessor directives, we do not possess a direct control over the runtime library. Additionally, since the objective of AL4SAN is to provide a unified API that can be dynamically processed at runtime, we resort to the open-source OpenMPLLVM library. This enables us to avail of the OpenMP task-based functionality in the AL4SAN library. Subsequently, we are able to add extra standard features [34] like "workspace" or "scratchpad" buffer per worker using AL4SAN_Workspace_Create. Ultimately, the objective is to leverage the $\mathrm{C}++$ language constructs, while incidentally providing data abstraction. The current support of LLVM anticipates the broader idea of a compiler-based approach 


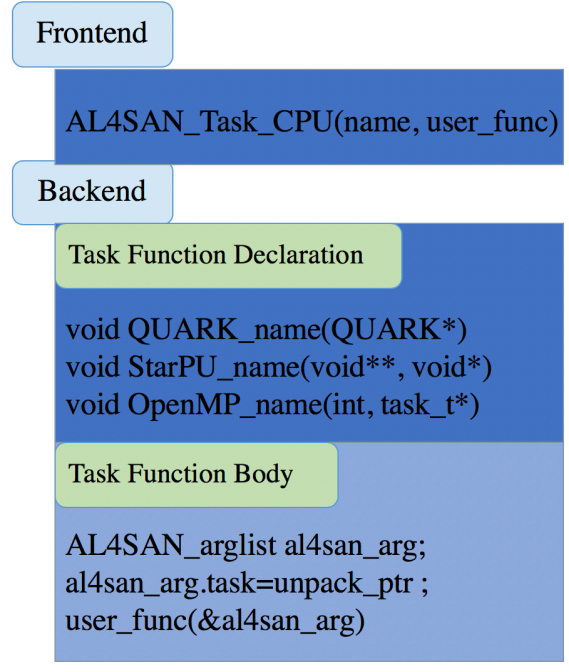

Fig. 3: AL4SAN task's work function.

for tasking, besides providing a hook to one of the most well-established programming models, i.e., OpenMP.

\subsection{Applicability of AL4SAN}

Although AL4SAN is in a continuous development, we here present its up-to-date status and slated developments. AL4SAN currently supports task queuing, task priorities, and task/data management for the considered runtime systems. It even features runtime-specific attributes to broaden its use cases. With regards to upcoming developments of the supported runtime systems, we have defined a systematic approach so that end users can add new runtime features directly onto their applications using AL4SAN APIs. This AL4SAN API simply updates the internal list of arguments to be passed on to the "unpack" runtime internal function. To achieve its runtime-agnostic goal, AL4SAN uses the AL4SAN_arglist API to take into account all runtime specific arguments and hands them over to the low-level "unpack" routines of each runtime. The "unpack" runtime routine parses the argument list and activates the proper runtime commands, accordingly. This feature enables to constantly catch up with the runtime system latest developments. For instance, if the underlying runtime supports private task memory per worker thread, AL4 SAN may leverage it and pass the workspace information to the corresponding runtime supporting such a feature.

AL4 SAN may encounter obvious bottlenecks with closedsource or commercial compilers for openMP runtime support, since the internal APIs are not exposed except through LLVM. But this concern is actually typical as it applies to any applications that rely on closed-source or commercial software packages. The application developer may always translate their AL4SAN-based code to the native OpenMP version, once the runtime assessment phase finishes.

\section{ACHIEVING RUNTIME INTEROPERABILITY WITH AL4SAN}

Runtime interoperability is an interesting mechanism because it seamlessly enables to assign the most appropriate task in terms of workload, hardware requirements and any user-specific criteria to the most relevant runtime system at runtime.

During AL4SAN installation, the desired runtimes can be chosen using the CMake building tool. At runtime, the user has then the capability of switching runtimes through the AL4SAN_Switch_Runtime API. This interface takes the runtime's alias as its first argument along with number of desired CPU cores and GPUs (if applicable) as its second and third arguments, respectively. AL4SAN context data structure keeps the state of the chosen system during the lifetime of the active runtime. AL4SAN high level API consults first AL4SAN context to identify the current runtime, then according to the user new preferred engine, it calls the API of the desired internal runtime system and carries on with the selected runtime system. This runtime switch requires a global synchronization in which the master thread is in charge of ensuring a safe relay between runtimes at runtime. This may engender overheads due to resource synchronizations in-between runtime switch. However, the runtime interoperability feature turns out to be a critical source of synergism across existing numerical libraries and their respective runtime systems. It permits to make the various runtime options coexist for the benefit of the applications.

To highlight the novelty of this feature, we introduce yet another workload which inherently contains, respectively, compute-bound and memory-bound computational phases. Thanks to AL4SAN, we deploy the first task-based generalized symmetric eigensolver (GSEVP) [35], [36] on GPUs. This represents an important numerical algorithm for material science applications, when solving the Shrödinger equation for electronic structure calculations [37]. The latest GSEVP implementations employ a multi-stage approach to increase hardware occupancy [38]-[42]. The compute-bound phase contains two steps. First, the generalized symmetric eigenvalue problem is transformed into a standard eigenvalue problem using a Cholesky factorization followed by a triangular solve (referred to Trans). Then, the resulting matrix is reduced to band form through successive orthogonal transformations (referred to Band). Last, the memorybound computational phase incurs in further reducing the symmetric band matrix to a condensed tridiagonal matrix via a Bulge-chasing procedure (referred to Bulge). The eigensolver (typically based on a divide and conquer algorithm), can then be applied on the tridiagonal matrix to extract the eigenspectrum of interests.

These three successive steps are only available in the PLASMA library with the QUARK runtime system, which supports only x86 CPUs. In particular, the implementation of the Bulge step makes tasks operate on data, which spans across several tiles. This is only possible with the QUARK runtime system and will require non-negligible development to make it compliant with all runtimes. Using the runtime interoperability feature of AL4SAN, we can accelerate the GSEVP implementation on GPUs using the StarPU runtime system for the first two compute-bound steps (Trans and Band) and carry on with QUARK for the memory-bound stage (Bulge). The resulting GSEVP can now be integrated into the Chameleon library, broadening significantly its algorithmic scope. 


\section{Performance Results}

In this section, we study the overhead of AL4SAN and compare it against the native task-based runtime version using various workloads. We show the performance breakdown of the main AL4SAN's user APIs and highlight the overall performance on several hardware configurations. Last but not least, we present the effectiveness of AL4SAN's runtime interoperability feature on a critical task-based dense matrix operation.

\subsection{Apparatus}

We conduct our overhead assessment and performance benchmarking campaign using the two workloads introduced in Section 4, i.e., compute-bound and memorybound, which can be found in the Chameleon (v1.0.0) and HiCMA (v0.1.0) libraries, respectively. To build the overall software stacks for both libraries, we use StarPU (v1.2.6), and QUARK (v2.8.0) compiled with GCC (v5.5.9). We also install the OpenMP-LLVM (v6.0.1) with the CLANG compiler. Moreover, Table 2 outlines the hardware specifications of the subsequent experiments: from single shared-memory multicore node, to system equipped with hardware accelerators, all the way up to the Shaheen-2 Cray XC40 distributedmemory supercomputer system. All computations are performed in double precision floating-point arithmetic and performance averages are reported out of five executions.

\subsection{Compute-Bound Workloads}

This subsection investigates the performance impact of the AL4SAN abstraction layer using task-based dense operations on the three systems under consideration with the four dynamic runtime systems. Figure 4 shows the performance impact of the AL4SAN abstraction layer using a task-based dense DGEMM operation on the three systems under consideration with the three dynamic runtime systems. Some runtimes may not support distributed-memory and/or GPU-based systems, and therefore, do not appear in the corresponding performance graphs. As expected, this compute intensive operations permits to hide the overhead not only of the native runtime but also of the AL4SAN abstraction layer on any considered runtime systems and hardware configurations. The obtained performance shows a decent percentage of the sustained peak performance. We also assess the performance overhead of RAJA [27] abstracting OpenMP's fork-join using C++ loop-oriented constructs. Since RAJA currently supports only independent tasks inferred by the fork-join paradigm, we only report results on DGEMM benchmark to have an apple-to-apple performance comparison. Figure 5 shows the overhead of RAJA as well as AL4SAN using OpenMP task directive for DGEMM. Although this may not be a fair comparison, the main take-home message of this experiment is just to demonstrate that the overheads of both abstraction layers are negligible on this compute-intensive workload.

Figure 6 shows the performance impact of task-based AL4SAN using a high-level dense linear algebra operation, i.e., the dense Cholesky factorization. Cholesky operation is not as embarrassingly parallel as DGEMM and requires the runtime system to schedule tasks, while making sure

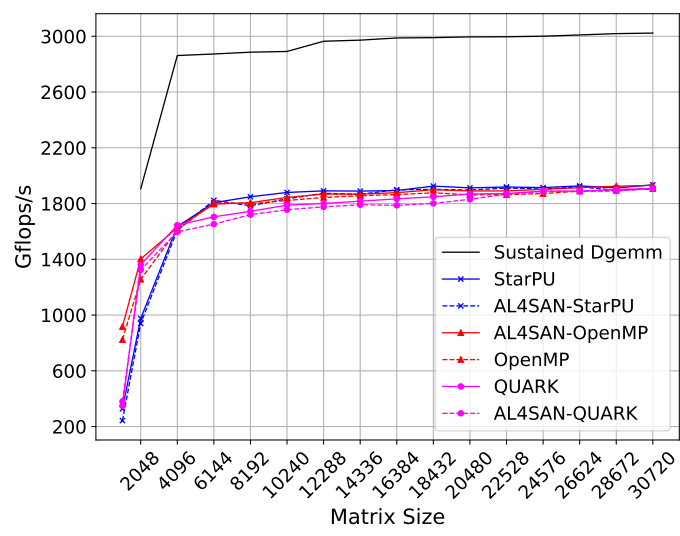

(a) Skylake.

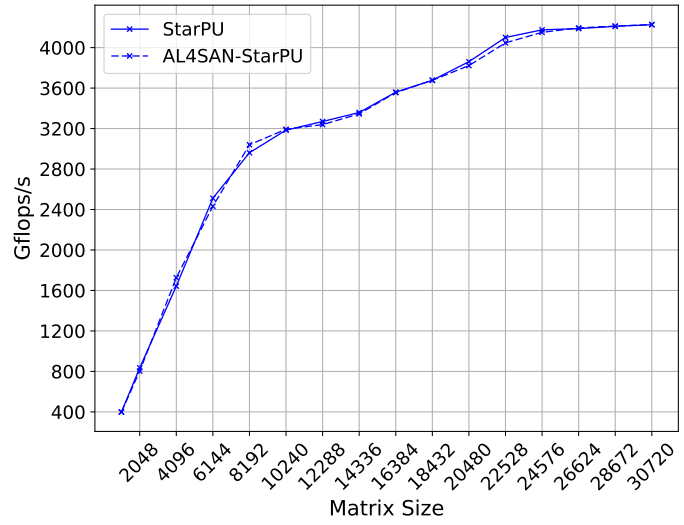

(b) Broadwell + 8 K80 NVIDIA GPUs.

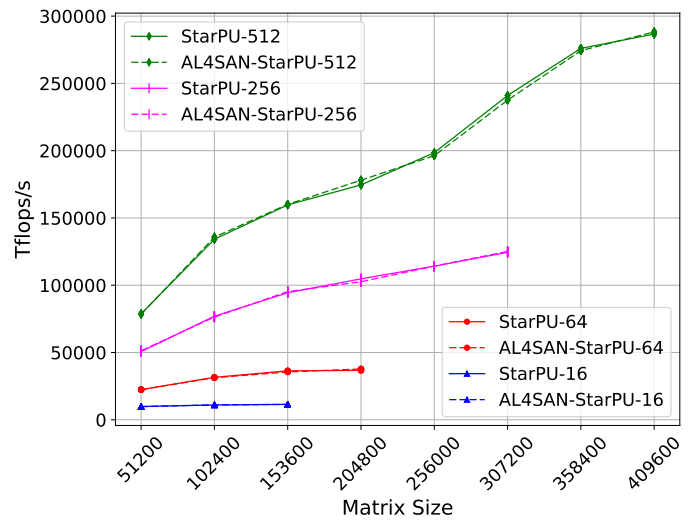

(c) Shaheen-2 scalability on 16, 64, 256 and 512 nodes.

Fig. 4: Performance impact of AL4SAN using task-based dense DGEMM.

the data dependencies are satisfied. Similarly to DGEMM workload, the overhead of AL4SAN is also negligible when using dense Cholesky factorization on all runtimes and system configurations.

\subsection{Memory-Bound Workloads}

This section provides an in-depth understanding of AL4SAN's impact on the performance of the underlying runtime system, when the workload is memory-bound. This may further expose the overhead engendered by any additional layers introduced in the software stack of the user applications. We perform experiments using the tile 
TABLE 2: Hardware specifications.

\begin{tabular}{|c|c|c|c|c|c|}
\hline & Shaheen-2 & Broadwell+GPUs & Broadwell+GPUs & Broadwell+GPUs & Skylake (SKL) \\
\hline Family & E5V3 & E5V4 & E5V4 & E5V4 & Scalable \\
\hline Model & 2698 & 2680 & 2698 & 2698 & 8176 \\
\hline Node(s) & 6144 & 1 & 1 & 1 & 1 \\
\hline Socket(s) & 2 & 2 & 2 & 2 & 2 \\
\hline Cores & 32 & 28 & 56 & 40 & 40 \\
\hline $\mathrm{GHz}$ & 2.60 & 2.40 & 2.20 & 2.20 & 2.10 \\
\hline Watts/socket & 135 & 120 & 135 & 135 & 165 \\
\hline DDR4 (GB) & 128 & 256 & 504 & 504 & 264 \\
\hline Max Turbo GHz & 3.40 & 3.40 & 3.60 & 3.60 & 3.80 \\
\hline Governor & performance & performance & performance & performance & on-demand \\
\hline Turbo Boost & available & available & available & available & available \\
\hline GPU Type & None & 8x Nvidia K80 & 8x Nvidia P100 & 8x Nvidia V100 & None \\
\hline
\end{tabular}

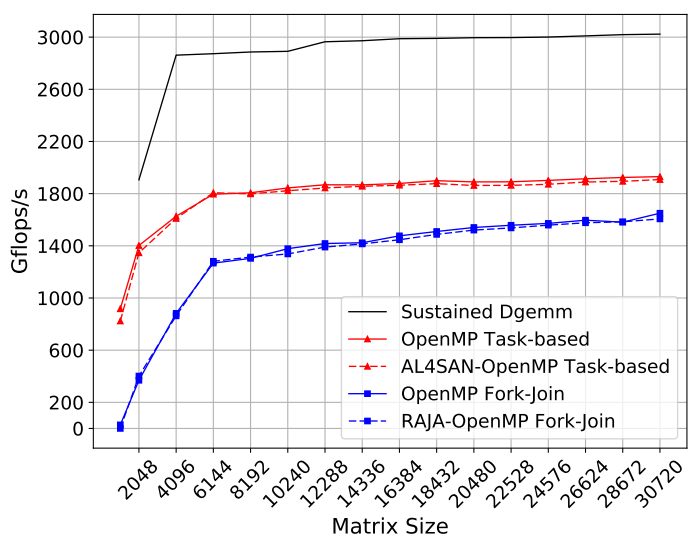

Fig. 5: OpenMP dense DGEMM with task-based AL4SAN and fork-join RAJA on Skylake.

low-rank Cholesky factorization from the HiCMA library on homogeneous systems, since the port of HiCMA on hardware accelerators is still work in progress. We inspect the distribution of task scheduling borrowing some benchmarking techniques from [43], [44] as well as the breakdown of the three AL4SAN abstraction routine categories, as explained in Section 3.

Impact on Task Scheduling Distribution. One of the crucial means for assessing the quality of a runtime's abstraction is quantifying how much it interacts with the scheduling behavior of the given runtime. This test is motivated by the fact that a major change in the distribution is likely to happen if options like "priority" and "locality" have to be compromised to maintain the abstraction's consistency. Therefore, we run the tile low-rank Cholesky with 200k matrix size on the Skylake system. We time the individual task scheduling latency when enabling and disabling AL4SAN, as shown in Figure 7. The x-axis represents the time in seconds, whereas the y-axis is the number of sample tasks that correspond to each specific time. From Figure 7 , we observe a small shift to the right of the $x$ axis on all studied runtimes, due to the overhead explained herein. However, the overall distribution is not affected by introducing AL4SAN, which confirms that it does not pose a problematic side-effect. We can still see that the bars do not completely overlap, which is understandable due to non-deterministic behavior of dynamic runtime systems. Hence, we apply the Two-sample Kolmogorov-Smirnov
(KSTest) [45]. This tests the decision for the null hypothesis that the data obtained from two runtime configurations belong to the same continuous distribution. It uses the maximum absolute difference between the two Cumulative Distribution Functions (CDF) and applies the following test statistic $D^{*}=\max _{x}\left(\left|\hat{F}_{1}\left(x_{1}\right)-\hat{F}_{2}\left(x_{2}\right)\right|\right)$. We apply KSTest to the datasets produced by experiments in Figure 7. The test approves the null hypothesis that $x_{1}$ and $x_{2}$ are from the same CDF with Alpha significance level of 0.01. We note that [45] does not directly mention the two-sample KStest, but our test is indeed both two-sided (alpha testing is divided between the two sides of the distribution) and two-sample test. Traditionally, the empirical data is compared to the theoretical Cumulative Distribution Function (CDF) whereas we compare two sample datasets to further verify AL4SAN's effect on the original distribution of the underlying runtime.

Execution Time. Figure 8 presents the performance impact of task-based AL4SAN using tile low-rank Cholesky factorization. Although there exists an overhead highlighted from the task scheduling distribution, the lightweight abstraction layer of AL4SAN limits it to a maximum of $10 \%$, regardless of the underlying task-based engines.

Overhead Analysis. Figure 9 further analyzes the overhead introduced by AL4SAN's abstraction that is shown by Figure 8. Each pair of bars represents the comparison with and without the use of AL4SAN abstraction. The y-axis is the cumulative time of all tasks, which is most likely asynchronous, but for the purpose of this experiment, the time is stacked up. "Task Insert" is the cumulative amount of elapsed time incorporating the task dependencies, scheduling options, etc., and eventually inserting it using the specific runtime's API. This results in an overhead because of the different ways the va_list has to be interpreted to be compatible with the corresponding Task Insertion backend API. "Task Unpack" reflects the task unpacking time, and it is expected to affect the performance since different runtimes tackle unpacking phase with different procedures, as described by the interface in Section 5 . These times may slightly vary due to the individual differences in matching the underlying runtime's API. The "Runtime Management" is measured from the moment the task is inserted until it is scheduled. This time is again tightly-coupled to the runtime. Hence, the small overhead contributed by AL4 SAN is mostly attributable to the scheduling options adopted by AL4SAN to unify the interfaces with minimal performance 


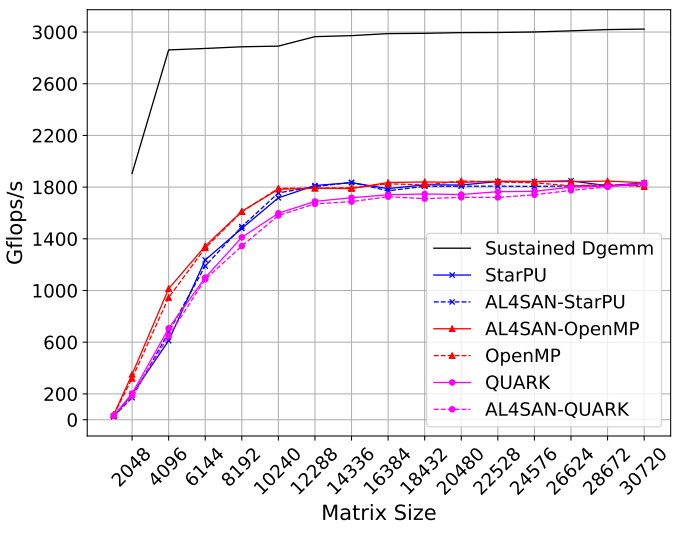

(a) Skylake.

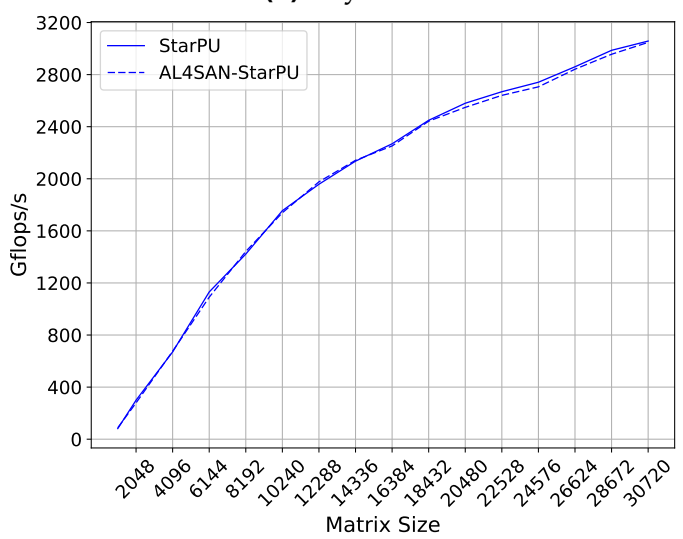

(b) Broadwell + 8 K80 NVIDIA GPUs.

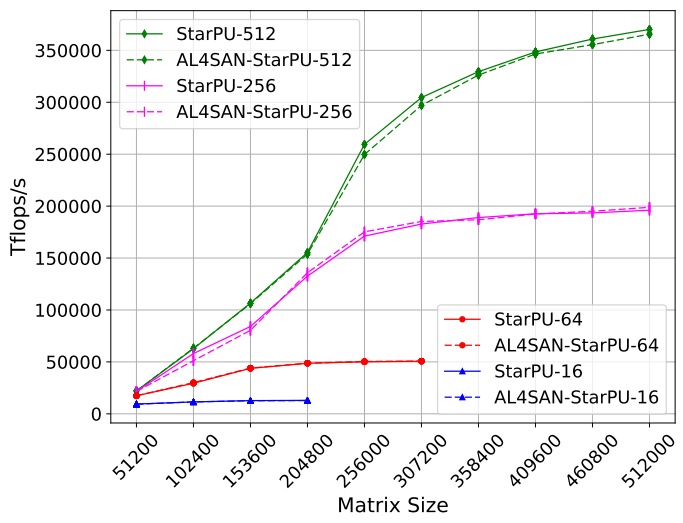

(c) Shaheen-2 scalability on 16, 64, 256 and 512 nodes.

Fig. 6: Performance impact of AL4SAN using task-based dense Cholesky factorization.

penalty. Moreover, Figure $9 \mathrm{~b}$ shows an overall increase in time because StarPU is configured with the least tile size, which yields more tasks by definition. In the OpenMP's experiment shown by Figure 9c, AL4SAN has the most overhead due to the non-existence of explicit calls to insert and unpack in the original OpenMP task API.

\subsection{Performance Improvement of the Generalized Sym- metric Eigenvalue Problem}

The Generalized Symmetric Eigenvalue Problem (GSEVP) corresponds to a much more challenging benchmark for

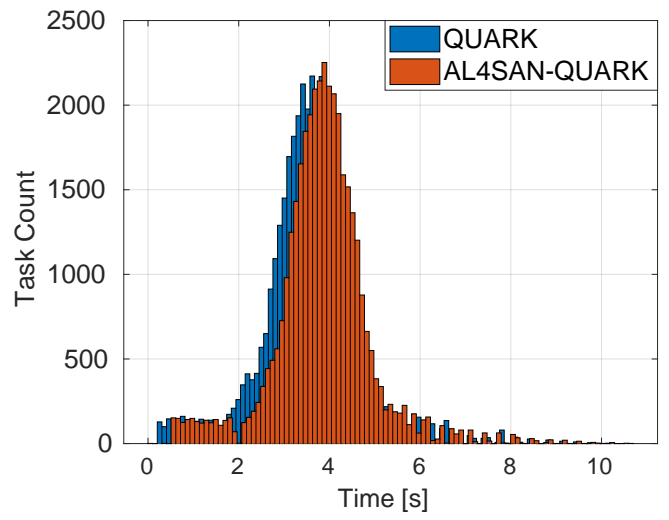

(a) QUARK.

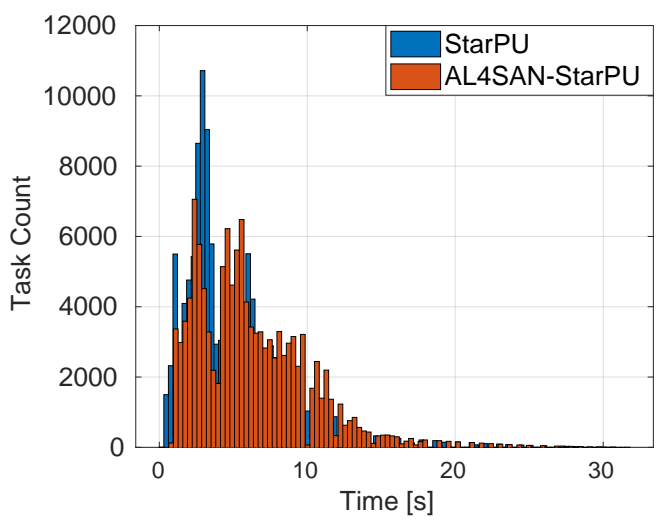

(b) Starpu.

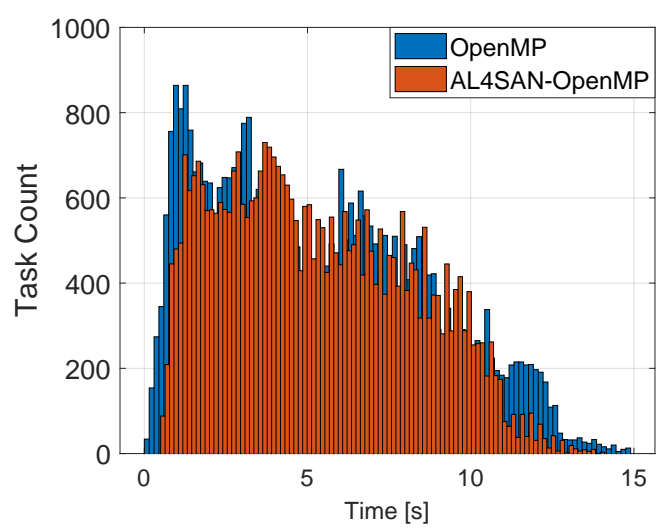

(c) OpenMP.

Fig. 7: Task scheduling distribution for tile low-rank Cholesky factorization.

AL4SAN. In fact, it is not just a benchmark for AL4SAN. It highlights how AL4SAN may permit to seamlessly deploy complex task-based algorithms by combining features from different runtime systems.

To illustrate the performance impact of switching runtimes at runtime on GESVP, Figure 10 shows the performance breakdown of its various computational steps, as described in Section 6, on a two-socket 14-core Intel Broadwell with four (Figure 10a) and eight (Figure 10b) NVIDIA K80 GPUs. The y-axis represents time in seconds, whereas the $\mathrm{x}$-axis shows different tile sizes for a $45 \mathrm{~K} \times 45 \mathrm{~K}$ matrix size. Each pair of bars compares AL4SAN (which combines 


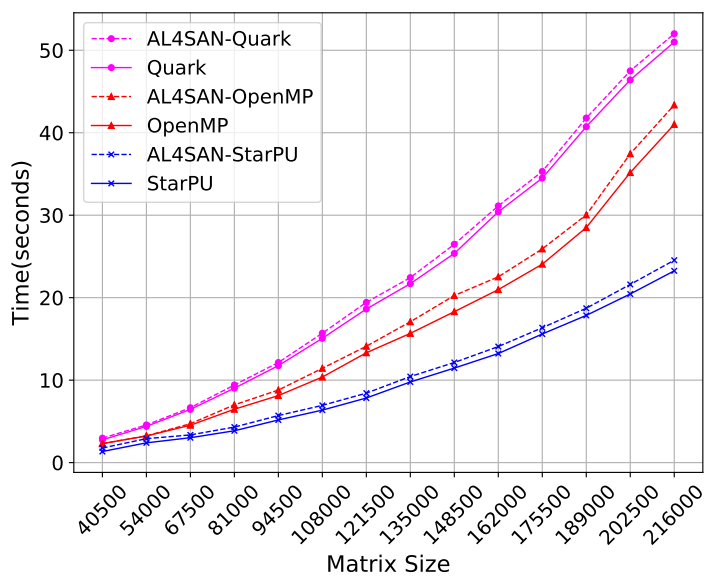

(a) Skylake.

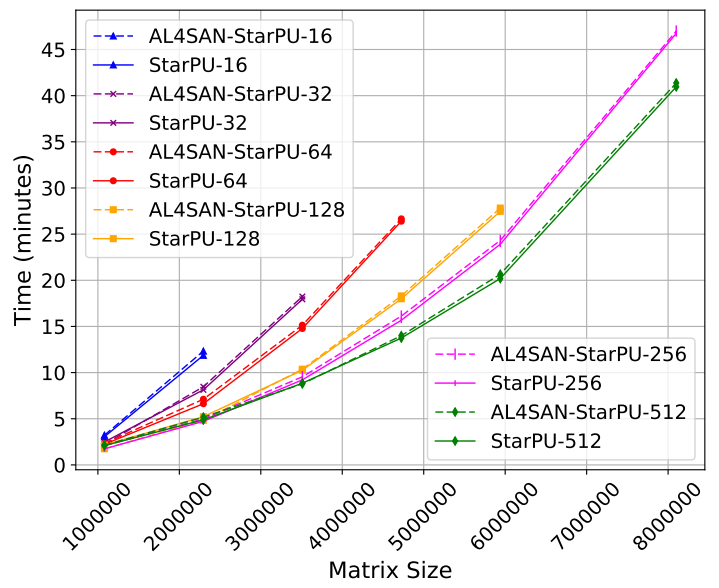

(b) Shaheen-2 scalability on 16, 32, 64, 128, 256 and 512 nodes.

Fig. 8: Performance impact of task-based AL4SAN using tile low-rank Cholesky factorization.

StarPU for GPU acceleration of the Trans and Band steps and QUARK for the Bulge step) against the native PLASMA based on QUARK for different phases of the algorithm. For each block size, the first two pair of bars show the total execution time, the second pair reports the elapsed time for Trans, the two subsequent bars show Band reduction stage, and last two bars represent Bulge-chasing stage.

AL4SAN outperforms PLASMA as we increase the block sizes, since it benefits from the compute-intensive steps running on GPUs with StarPU runtime system as backend for AL4SAN. However, using small tile sizes, this creates too many small tiles to be moved between CPU and GPU, which produces negative impact on the overall performance that StarPU is not able to cope with. This affects AL4SAN performance and makes it run slower than PLASMA.

AL4SAN increases performance of the memory-bound Bulge stage by reducing the overhead due to bus bandwidth saturation. Indeed, the Bulge-chasing stage inherently necessitates large-strided memory access, which saturates the memory bandwidth with a few active threads. Thanks to the possibility to decrease the number of threads during the runtime switch, AL4SAN mitigates the overhead of false sharing and enhances the overall Bulge-chasing performance. Moving from four to eight GPUs permits to im-

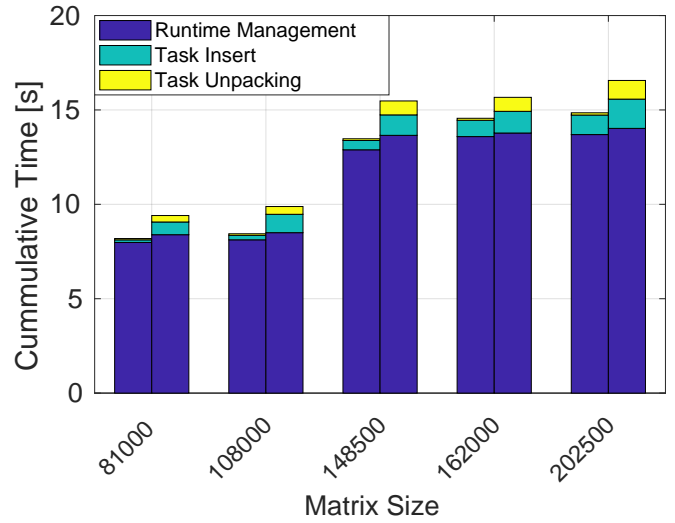

(a) Left: QUARK, right: AL4 SAN-QUARK.

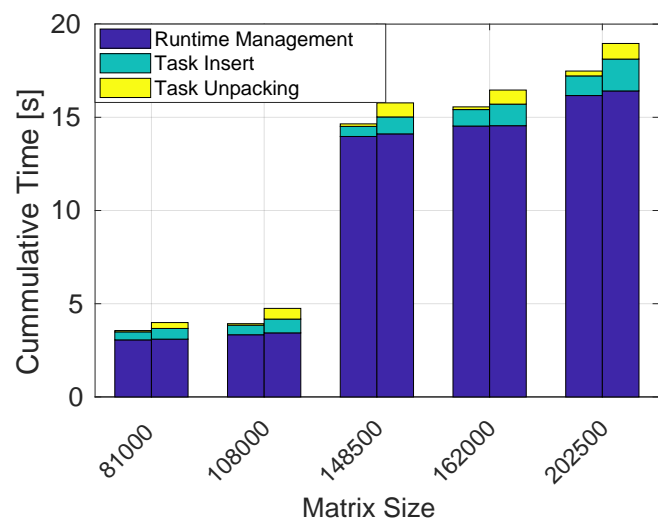

(b) Left: StarPU, right: AL4 SAN-StarPU.

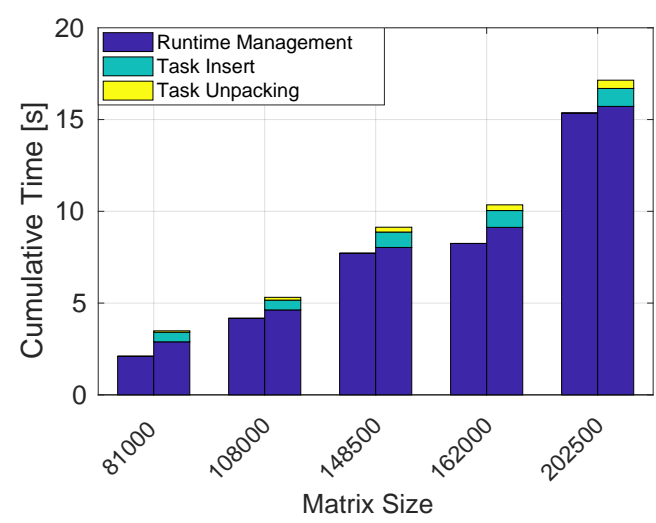

(c) Left: OpenMP, right: AL4SAN-OpenMP.

Fig. 9: Overhead breakdown across runtimes on Skylake.

prove the compute-bound computational phases (Trans and Band), especially when dealing with large tile sizes. Hence, the GSEVP implementation with AL4SAN outperforms the native PLASMA implementation by more than $35 \%$.

Figure 11 evaluates the overall GSEVP performance based on AL4SAN and compares it to ELPA [46] and PLASMA [1] on GPU-based systems equipped with three generations of accelerators. We use fewer threads for ELPA to activate the canonical algorithm by satisfying the restriction of a square grid of processes. PLASMA runs on all x86 cores available on the hardware system. AL4SAN adopts a flexible resource allocation to maximize performance: StarPU needs one thread per GPU to manage data motion 


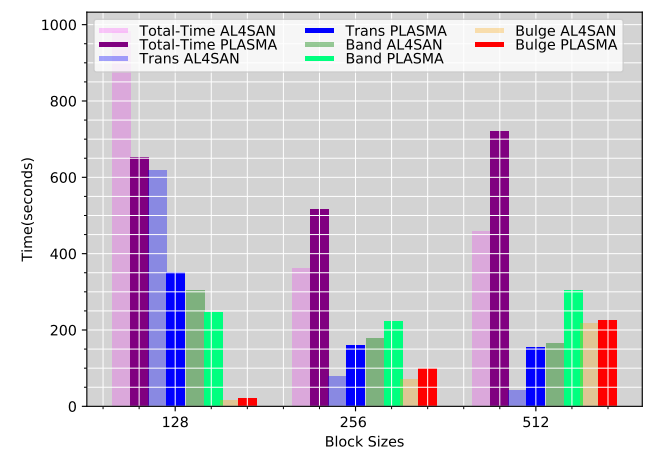

(a) AL4 SAN versus PLASMA using four K80 GPUs.

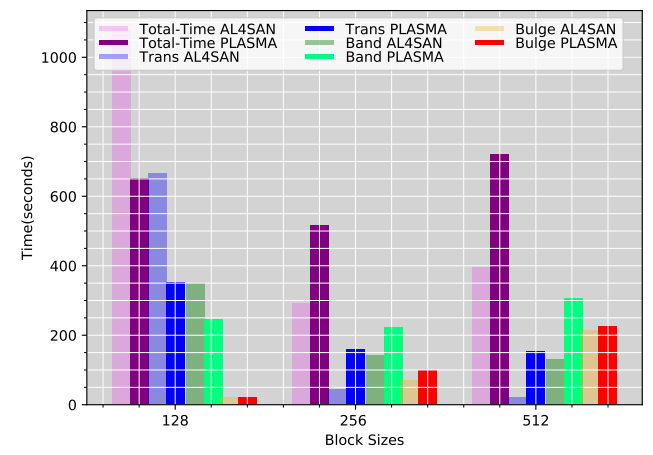

(b) AL4 SAN versus PLASMA using eight K80 GPUs.

Fig. 10: Performance breakdown analysis for three-steps task-based generalized symmetric eigenvalue problem using AL4SAN (StarPU and QUARK) and PLASMA (QUARK only) on a $45 \mathrm{~K} \times 45 \mathrm{~K}$ matrix size using a two-socket 14 -core Intel Broadwell equipped with K80 NVIDIA GPUs.

between CPU and GPU during the Trans and Band steps while QUARK uses less number of threads for the Bulge step. All reported hardware resources correspond to the best achieved performance for ELPA, PLASMA, and AL4SAN. AL4 SAN outperforms both GSEVP implementations, achieving almost a twofold speedup against ELPA on the system equipped with eight V100s, i.e., the latest GPU generations. Moving from K80 to V100 GPUs, the GSEVP implementation based on AL4SAN improved nearly by $33 \%$.

Although these results focus only on shared-memory systems with GPUs, AL4SAN may be deployed on stateof-the-art sparse eigensolvers, such as PFEAST [47] and Trilinos [48], which translates solving large sparse eigenvalue problems into solving small dense eigensolvers, which fit into single shared-memory nodes. Leveraging the GSEVP implementation to distributed-memory systems with AL4SAN will necessitate making the Bulge step compliant with runtimes beyond QUARK. This is an interesting research avenue but beyond the scope of this paper.

\section{Conclusion and Future Work}

This paper introduces the AL4SAN software library, an abstraction layer for standardizing APIs of task-based engines. AL4SAN currently supports three representative task-based

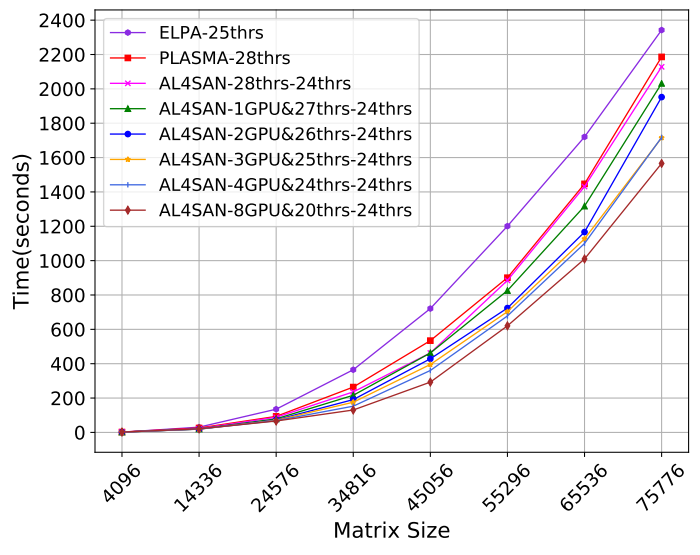

(a) Intel Broadwell + K80 NVIDIA GPUs.

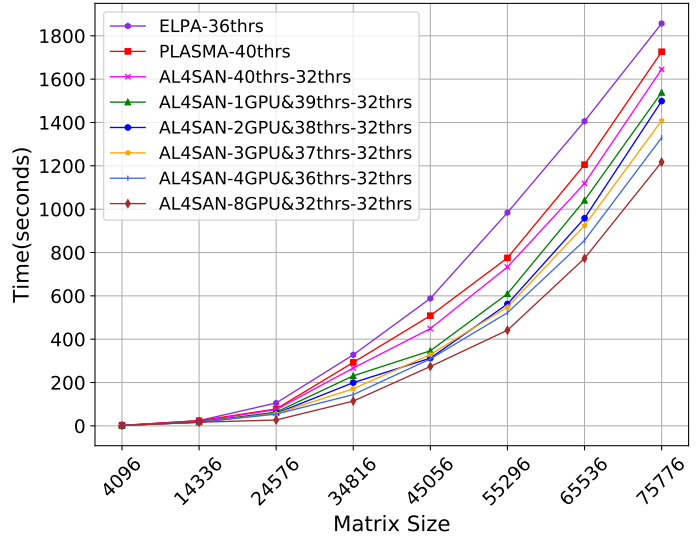

(b) Intel Broadwell + 8 P100 NVIDIA GPUs.

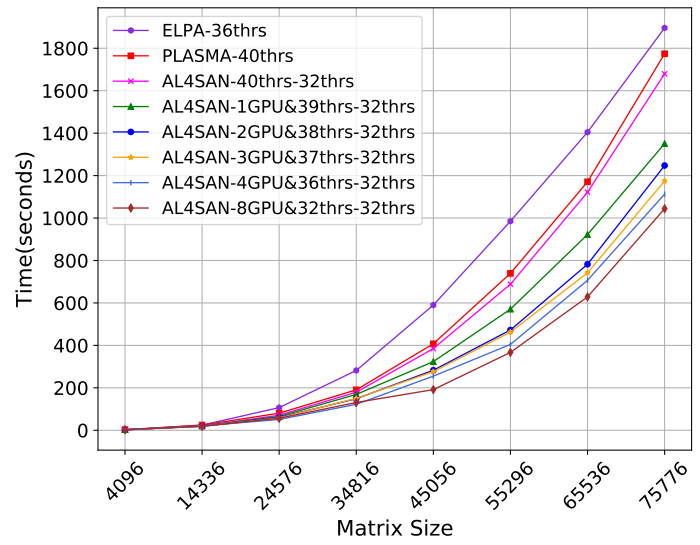

(c) Intel Broadwell + 8 V100 NVIDIA GPUs.

Fig. 11: Performance comparison of a three-steps task-based generalized eigenvalue problem using AL4SAN, ELPA, and PLASMA.

runtime systems relying on compiler technologies with language constructs and user-defined APIs, i.e., OpenMP, StarPU, and QUARK. AL4SAN enhances user-productivity since it permits to have a single application code running on top of various backend engines. Thanks to its lightweight abstraction, the overhead of AL4SAN is acceptable (less than $10 \%$ ), even in situations where the user application is memory-bound. Moreover, we have demonstrated how runtime interoperability may be a key feature in improving 
performance of existing task-based numerical algorithms. In particular, we have shown how the generalized symmetric eigensolver can benefit from the novel feature of blending two runtimes, achieving almost a twofold speedup against state-of-the-art eigensolvers. AL4SAN has been successfully integrated into the Chameleon [18] and HiCMA [19] libraries and is freely available at https://github.com/ecrc/al4san. Moving forward, we plan to extend the scope of AL4SAN with more runtimes, while further leveraging task and data abstraction through $\mathrm{C}++$ constructs.

\section{ACKNOWLEDGMENTS}

The authors would like to thank Kadir Akbudak from ECRC at King Abdullah University of Science and Technology (KAUST) and Mathieu Faverge and Florent Pruvost from INRIA Bordeaux for their support in integrating AL4SAN into the HiCMA and Chameleon libraries, respectively. The authors would like also to thank Cray Inc. and Intel in the context of the Cray Center of Excellence and Intel Parallel Computing Center awarded to ECRC at KAUST. For computer time, this research used Shaheen-2 supercomputer hosted at the Supercomputing Laboratory at KAUST.

\section{REFERENCES}

[1] E. Agullo, J. Demmel, J. Dongarra, B. Hadri, J. Kurzak, J. Langou, H. Ltaief, P. Luszczek, and S. Tomov, "Numerical Linear Algebra on Emerging Architectures: The PLASMA and MAGMA Projects," Journal of Physics: Conference Series, vol. 180, 2009.

[2] E. Agullo, O. Aumage, M. Faverge, N. Furmento, F. Pruvost, M. Sergent, and S. Thibault, "Achieving High Performance on Supercomputers with a Sequential Task-based Programming Model," IEEE Trans. Parallel Distributed Systems, 2017.

[3] R. Kriemann, "H-LU factorization on many-core systems," Computing and Visualization in Science, vol. 16, no. 3, 2013.

[4] J. Kurzak, P. Wu, M. Gates, I. Yamazaki, P. Luszczek, G. Ragghianti, and J. Dongarra, "SLATE Working Note 3: Designing SLATE: Software for Linear Algebra Targeting Exascale," Technical Report ICLUT-17-06, Innovative Computing Laboratory, University of Tennessee, 2017.

[5] G. Bosilca, A. Bouteiller, A. Danalis, M. Faverge, A. Haidar, T. Herault, J. Kurzak, J. Langou, P. Lemarinier, H. Ltaief, P. Luszczek, A. YarKhan, and J. Dongarra, "Flexible Development of Dense Linear Algebra Algorithms on Massively Parallel Architectures with DPLASMA," in IPDPS Workshops. IEEE, 2011.

[6] X. Lacoste, M. Faverge, G. Bosilca, P. Ramet, and S. Thibault, "Taking Advantage of Hybrid Systems for Sparse Direct Solvers via Task-Based Runtimes," in 2014 IEEE International Parallel $\mathcal{E}$ Distributed Processing Symposium Workshops (IPDPSW), vol. 00, May 2014.

[7] E. Agullo, A. Buttari, A. Guermouche, and F. Lopez, "Task-Based Multifrontal QR Solver for GPU-Accelerated Multicore Architectures," in 22nd IEEE International Conference on High Performance Computing (HiPC). IEEE Computer Society, 2015.

[8] H. Jagode, A. Danalis, and J. Dongarra, "Accelerating NWChem Coupled Cluster Through Dataflow-Based Execution," The International Journal of High Performance Computing Applications, 01-2017 2017.

[9] H. Ltaief, D. Gratadour, A. Charara, and E. Gendron, "Adaptive Optics Simulation for the World's Largest Telescope on Multicore Architectures with Multiple GPUs," in Proceedings of the Platform for Advanced Scientific Computing Conference, ser. PASC '16. New York, NY, USA: ACM, 2016.

[10] L. A. Berry, W. R. Elwasif, J. M. Reynolds-Barredo, D. Samaddar, R. Sánchez, and D. E. Newman, "Event-Based Parareal: A DataFlow Based Implementation of Parareal," Journal of Computational Physics, vol. 231, no. 17, 2012.
[11] Q. Meng, A. Humphrey, J. Schmidt, and M. Berzins, "Investigating Applications Portability with the Uintah DAG-based Runtime System on PetaScale Supercomputers," in Proceedings of the International Conference on High Performance Computing, Networking, Storage and Analysis, ser. SC '13. New York, NY, USA: ACM, 2013.

[12] P. Thoman, K. Dichev, T. Heller, R. Iakymchuk, X. Aguilar, K. Hasanov, P. Gschwandtner, P. Lemarinier, S. Markidis, H. Jordan et al., "A taxonomy of task-based parallel programming technologies for high-performance computing," The Journal of Supercomputing, vol. 74, no. 4, pp. 1422-1434, 2018.

[13] A. Yarkhan, "Dynamic Task Execution on Shared and Distributed Memory Architectures," PhD Dissertation, no. December, 2012.

[14] C. Augonnet, S. Thibault, R. Namyst, and P.-A. Wacrenier, "StarPU: A Unified Platform for Task Scheduling on Heterogeneous Multicore Architectures," Concurrency and Computation: Practice and Experience, vol. 23, no. 2, 2011.

[15] OpenMP, “OpenMP 4.0 Complete Specifications," 2013.

[16] A. Duran, R. Ferrer, E. Ayguade, R. M. Badia, and J. Labarta, "A Proposal to Extend the OpenMP Tasking Model with Dependent Tasks," International Journal of Parallel Programming, vol. 37, no. 3, 2009.

[17] OpenMP, “OpenMP 3.0 Complete Specifications," 2008.

[18] "The Chameleon Project," https://gitlab.inria.fr/solverstack/ chameleon, INRIA Bordeaux, 2019.

[19] "The HiCMA Library," http://github.com/ecrc/hicma, Extreme Computing Research Center, King Abdullah University of Science and Technology, 2019.

[20] K. Akbudak, H. Ltaief, A. Mikhalev, A. Charara, A. Esposito, and D. Keyes, "Exploiting Data Sparsity for Large-Scale Matrix Computations," in European Conference on Parallel Processing, vol. 11014. Springer, 2018.

[21] M. P. Robson, R. Buch, and L. V. Kale, "Runtime Coordinated Heterogeneous Tasks in Charm++," in Proceedings of the Second Internationsl Workshop on Extreme Scale Programming Models and Middleware, ser. ESPM2. Piscataway, NJ, USA: IEEE Press, 2016.

[22] M. Bauer, S. Treichler, E. Slaughter, and A. Aiken, "Legion: Expressing locality and independence with logical regions," in International Conference for High Performance Computing, Networking, Storage and Analysis, SC, 2012.

[23] H. C. Edwards, C. R. Trott, and D. Sunderland, "Kokkos: Enabling Manycore Performance Portability Through Polymorphic Memory Access Patterns," Journal of Parallel and Distributed Computing, vol. 74, no. 12, 2014.

[24] J. Bueno, J. Planas, A. Duran, R. M. Badia, X. Martorell, E. Ayguade, and J. Labarta, "Productive Programming of GPU Clusters with OmpSs," Proceedings of the 2012 IEEE 26th International Parallel and Distributed Processing Symposium, IPDPS 2012, 2012.

[25] R. Al-Omairy, G. Miranda, H. Ltaief, R. M. Badia, X. Martorell, J. Labarta, and D. Keyes, "Dense matrix computations on numa architectures with distance-aware work stealing," Supercomputing Frontiers and Innovations, vol. 2, no. 1, pp. 49-72, 2015.

[26] T. Heller, H. Kaiser, and K. Iglberger, "Application of the ParalleX Execution Model to Stencil-Based Problems," Computer Science Research and Development, vol. 28, no. 2-3, 2013.

[27] R. Hornung, H. Jones, J. Keasler, R. Neely, O. Pearce, S. Hammond, C. Trott, P. Lin, C. Vaughan, J. Cook, R. Hoekstra, B. Bergen, J. Payne, and G. Womeldorff, "RAJA," Lawrence Livermore National Laboratory, ASC Tri-lab Co-design Level 2 Milestone Technical Report LLNL-TR-677453, 2015.

[28] J. Dokulil, M. Sandrieser, and S. Benkner, "Implementing the Open Community Runtime for Shared-Memory and DistributedMemory Systems," Proceedings - 24th Euromicro International Conference on Parallel, Distributed, and Network-Based Processing, PDP 2016, 2016

[29] J. Bennett, R. Clay, G. Baker, M. Gamell, D. Hollman, S. Knight, H. Kolla, G. Sjaardema, N. Slattengren, K. Teranishi et al., "Asynchronous Many-Task Runtime System Analysis and Assessment for Next Generation Platforms," Sandia National Laboratory, Technical Report SAND2015-8312, 2015.

[30] C. Newburn, "Hihat: A new way forward for hierarchical heterogeneous async tasking," Distributed and Heterogeneous Programming in $\mathrm{C} / \mathrm{C}++$ Workshop, (Presentation), May 2017. [Online]. Available: https://www.iwocl.org/wp-content/ uploads/dhpcc17-cj-hihat.pdf

[31] W. Hackbusch, Hierarchical matrices: Algorithms and Analysis. Springer, 2015, vol. 49. 
[32] P. Virouleau, P. Brunet, F. Broquedis, N. Furmento, S. Thibault, O. Aumage, and T. Gautier, "Evaluation of OpenMP Dependent Tasks with the KASTORS Benchmark Suite," in International Workshop on OpenMP. Springer, 2014.

[33] A. Danalis, G. Bosilca, A. Bouteiller, T. Herault, and J. Dongarra, "PTG: An Abstraction for Unhindered Parallelism," Proceedings of WOLFHPC 2014: 4th International Workshop on Domain-Specific Languages and High-Level Frameworks for High Performance Computing - Held in Conjunction with SC 2014: The International Conference for High Performance Computing, Networking, Stor, 2014.

[34] A. YarKhan, J. Kurzak, P. Luszczek, and J. Dongarra, "Porting the Plasma Numerical Library to the OpenMP Standard," International Journal of Parallel Programming, vol. 45, no. 3, 2017.

[35] G. H. Golub and C. F. Van Loan, Matrix Computations, 3rd ed., ser John Hopkins Studies in the Mathematical Sciences. Baltimore, Maryland: Johns Hopkins University Press, 1996.

[36] L. N. Trefethen and D. Bau, Numerical Linear Algebra. Philadelphia, PA: SIAM, 1997. [Online]. Available: http://www.siam.org/books/OT50/Index.htm

[37] Y. Saad, A. Stathopoulos, J. Chelikowsky, K. Wu, and S. Ögüt, "Solution of large eigenvalue problems in electronic structure calculations," BIT Numerical Mathematics, vol. 36, no. 3, pp. 563-578, Sep 1996. [Online]. Available: https: //doi.org/10.1007/BF01731934

[38] B. Lang, "Efficient eigenvalue and singular value computations on shared memory machines," Parallel Computing, vol. 25, no. 7, pp. 845-860, 1999.

[39] C. H. Bischof, B. Lang, and X. Sun, "Algorithm 807: The SBR Toolbox-Software for Successive Band Reduction," ACM Transactions on Mathematical Software, vol. 26, no. 4, pp. 602-616, 2000.

[40] A. Haidar, H. Ltaief, and J. Dongarra, "Parallel reduction to condensed forms for symmetric eigenvalue problems using aggregated fine-grained and memory-aware kernels," in Proceedings of 2011 International Conference for High Performance Computing, Networking, Storage and Analysis. ACM, 2011, p. 8.

[41] H. Ltaief, P. Luszczek, A. Haidar, J. J. Dongarra, and T. Ridge, "Solving the generalized symmetric eigenvalue problem using tile algorithms on multicore architectures." in PARCO, 2011, pp. 397404.

[42] P. Luszczek, H. Ltaief, and J. Dongarra, “Two-stage tridiagonal reduction for dense symmetric matrices using tile algorithms on multicore architectures," in 2011 IEEE International Parallel $\mathcal{E}$ Distributed Processing Symposium. IEEE, 2011, pp. 944-955.

[43] M. Abduljabbar, M. Al Farhan, R. Yokota, and D. Keyes, "Performance evaluation of computation and communication kernels of the fast multipole method on intel manycore architecture," in European Conference on Parallel Processing, vol. 10417. Springer, 2017, pp. 553-564.

[44] M. Abduljabbar, G. S. Markomanolis, H. Ibeid, R. Yokota, and D. Keyes, "Communication reducing algorithms for distributed hierarchical n-body problems with boundary distributions," in International Supercomputing Conference, vol. 10266. Springer, 2017, pp. 79-96.

[45] R. Simard, P. L'Ecuyer et al., "Computing the Two-Sided Kolmogorov-Smirnov Distribution," Journal of Statistical Software, vol. 39, no. 11, 2011

[46] A. Marek, V. Blum, R. Johanni, V. Havu, B. Lang, T. Auckenthaler, A. Heinecke, H. Bungartz, and H. Lederer, "The ELPA Library: Scalable Parallel Eigenvalue Solutions for Electronic Structure Theory and Computational Science," J Phys Condens Matter, vol. 26, no. 21, 2014. [Online]. Available: http: //www.ncbi.nlm.nih.gov/pubmed/24786764

[47] J. Kestyn, V. Kalantzis, E. Polizzi, and Y. Saad, "Pfeast: A high performance sparse eigenvalue solver using distributed-memory linear solvers," in SC '16: Proceedings of the International Conference for High Performance Computing, Networking, Storage and Analysis, Nov 2016, pp. 178-189.

[48] "Anasazi," https://trilinos.org/packages/anasazi/, Trilinos, 2019.

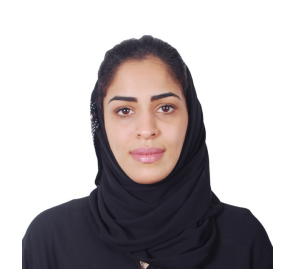

Rabab Alomairy is a computer science PhD student working in the Extreme Computing Research Center at King Abdullah University of Science and Technology (KAUST). Rabab earned her BSc degree in computer science from King Abdulaziz University and MSc degree from KAUST. Her research is centered around taskbased numerical libraries and applications, performance optimizations for multicore/manycore architectures and hardware accelerators, dynamic runtime systems, and API standardization. She is the president of SIAM student chapter at KAUST.

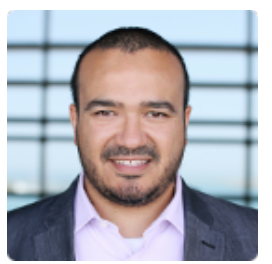

Hatem Ltaief is a Senior Research Scientist in the Extreme Computing Research Center at KAUST, where is also advising several KAUST students in their MS and PhD research. Hatem received the engineering degree from PolyTech Lyon at the University of Claude Bernard Lyon I, France, the MSc in applied mathematics at the University of Houston, and the $\mathrm{PhD}$ degree in computer science from the University of Houston. From 2008 to 2010, he was a Research Scientist in the Innovative Computing Laboratory in the Department of Electrical Engineering and Computer Science at the University of Tennessee, Knoxville. Hatem was awarded the NVIDIA GPU Research Center in 2012 (PI), the Intel Parallel Computing Center (co-PI) in 2015 and the Cray Center of Excellence (co-PI) in 2015. His research interests include parallel numerical algorithms, parallel programming models, and performance optimizations for multicore architectures and hardware accelerators.

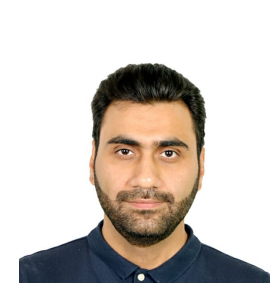

Mustafa Abduljabbar is a postdoctoral researcher at the Chalmers University of Technology, Sweden. He received his BSc degree in computer engineering from the American University of Sharjah, in 2008, and the PhD degree in computer science from the King Abdullah University of Science and Technology (KAUST). His research interests include parallel numerical solvers, scalability of the fast multipole method, performance trends of data-level and task-level parallel applications on Multi/Manycore hardware, and low-energy scheduling for parallel runtime systems.

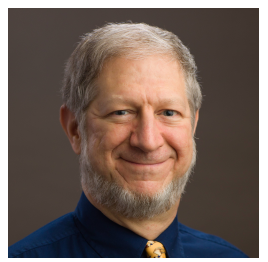

David E. Keyes received the BSE degree in aerospace and mechanical sciences from Princeton University, in 1978, and the PhD degree in applied mathematics from Harvard University, in 1984. He directs the Extreme Computing Research Center at KAUST. He works at the interface between parallel computing and the numerical analysis of PDEs, with a focus on scalable implicit solvers. He helped develop and popularize the Newton-Krylov-Schwarz (NKS), Additive Schwarz Preconditioned Inexact Newton (ASPIN), and Algebraic Fast Multipole (AFM) methods. Before joining KAUST as a founding dean in 2009, he led multi-institutional projects researching scalable solver software in the SciDAC and ASCI programs of the US DOE, ran University collaboration programs at LLNL's ISCR and NASA's ICASE, and taught at Columbia, Old Dominion, and Yale Universities. He is a fellow of SIAM, AMS, and the AAAS, and has been awarded the ACM Gordon Bell Prize, the IEEE Sidney Fernbach Award, and the SIAM Prize for Distinguished Service to the Profession. 